\title{
In vitro hepatic maturation of human embryonic stem cells by using a mesenchymal cell line derived from murine fetal livers.
}

\section{AUTHOR(S):}

Ishii, Takamichi; Yasuchika, Kentaro; Fukumitsu, Ken; Kawamoto, Tatsuya; Kawamura-Saitoh, Miho; Amagai, Yuji; Ikai, Iwao; ... Kawase, Eihachiro; Suemori, Hirofumi; Nakatsuji, Norio

\section{CITATION:}

Ishii, Takamichi ... [et al]. In vitro hepatic maturation of human embryonic stem cells by using a mesenchymal cell line derived from murine fetal livers.. Cell and tissue research 2010, 339(3): 505-512

\section{ISSUE DATE:}

2010-03

\section{URL:}

http://hdl.handle.net/2433/128863

\section{RIGHT:}

The original publication is available at www.springerlink.com; This is not the published version. Please cite only the published version.; この 論文は出版社版でありません。引用の際には出版社版をご確認ご利用 ください。 
Title: In vitro hepatic maturation of human embryonic stem cells using a mesenchymal cell line derived from murine fetal livers.

\section{Authors:}

Takamichi Ishii $^{1,2}$, Kentaro Yasuchika ${ }^{2}$, Ken Fukumitsu ${ }^{1,2}$, Tatsuya Kawamoto ${ }^{3}$, Miho Kawamura-Saitoh $^{3}$, Yuji Amagai ${ }^{3}$, Iwao Ikai ${ }^{2,4}$, Shinji Uemoto ${ }^{2}$, Eihachiro Kawase ${ }^{5}$, Hirofumi Suemori ${ }^{1}$, and Norio Nakatsuji ${ }^{5,6}$

\section{Affiliations:}

1. Laboratory of Embryonic Stem Cell Research, Stem Cell Research Center, Institute for Frontier Medical Sciences, Kyoto University

2. Department of Surgery, Graduate School of Medicine Kyoto University

3. Stem Cell and Drug Discovery Institute

4. Department of Surgery, Kyoto Medical Center, National Hospital Organization

5. Department of Development and Differentiation, Institute for Frontier Medical

Sciences, Kyoto University

6. Institute for Integrated Cell-Material Science, Kyoto University

\section{Financial support:}

This work was supported in part by grants from the New Energy and Industrial Technology Development Organization (NEDO) and the Scientific Research Fund of the Ministry of Education, Culture, Sports, Science, and Technology of Japan.

\section{Contact information:}

Takamichi Ishii, M.D., Ph.D.

53 Kawahara-cho Shogoin, Sakyo-ku, Kyoto, 606-8507, Japan

Telephone: +81-75-751-3821,

Fax: +81-75-751-3890,

E-mail: taishii@kuhp.kyoto-u.ac.jp 


\section{Abstract}

Hepatocytes derived from human embryonic stem cells (hESCs) are an attractive cell source for regenerative medicine. We previously reported the differentiation of hESCs into alpha-fetoprotein (AFP)-producing endodermal cells using extracellular matrix and growth factors. Moreover, we also reported the establishment of the MLSgt20 cell line, which was derived from mesenchymal cells residing in murine fetal livers and accelerated the hepatic maturation of both murine hepatic progenitor cells and murine ESCs. In this study, hESC-derived AFP-producing cells were isolated using a flow cytometer and co-cultured with MLSgt20 cells. The co-cultured hESC-derived AFP-producing cells had the immunocytologic characteristics of hepatocytes, expressed mature hepatocyte-markers by RT-PCR, and displayed higher hepatocyte functions, including ammonia removal ability, cytochrome P450 3A4/7 activity, and the ability to produce and store glycogen. However, the MLSgt20 cells did not directly cause undifferentiated hESCs to mature into hepatocyte-like cells. In conclusion, the co-culture method was successfully shown to differentiate hESC-derived endodermal 
cells into functional hepatocyte-like cells.

Key words: ESC, AFP, hepatocyte, MLSgt20, CYP3A 


\section{Introduction}

Hepatocytes derived from human embryonic stem cells (hESCs) are a

potential cell source for cell transplantation, bio-artificial livers, and drug discovery systems (Cai, et al., 2007, D'Amour, et al., 2005, Duan, et al., 2007). However, it has been difficult to obtain fully functional hepatocytes from hESCs, partly because there is still only limited knowledge of hepatic developmental biology and molecular mechanisms (Chen, et al., 2007, Ek, et al., 2007, Schuldiner, et al., 2000).

We previously reported that mesenchymal cells residing in murine fetal livers $(\mathrm{CD} 45-\mathrm{CD} 49 \mathrm{f} \pm \mathrm{Thy} 1+\mathrm{gp} 38+$ cells $)$ promoted hepatic maturation of hepatic progenitor cells (HPCs) (Hoppo, et al., 2004, Kamo, et al., 2007) and murine ESC-derived alpha-fetoprotein (AFP)-producing cells (Ishii, et al., 2005). Moreover, we successfully established the MLSgt20 cell line from CD45-CD49f \pm Thy $1+$ gp38+ murine fetal liver mesenchymal cells (Fukumitsu, et al., 2009). The MLSgt20 cells promoted the maturation of both murine HPCs and murine ESC-derived AFP-producing cells into functional hepatocyte-like cells. We also reported an effective method for the differentiation of hESCs into AFP-producing endodermal cells using growth factors and 
extracellular matrix (Ishii, et al., 2008). A hESC line expressing enhanced green fluorescent protein (EGFP) under the control of the human AFP enhancer/promoter was generated and differentiated into AFP-EGFP-positive cells with approximately $20 \%$ efficiency. However, it was technically difficult to further differentiate the hESC-derived AFP-producing cells into functional hepatocytes.

In the present study, we sought to make it possible for hESC-derived AFP-producing cells to mature into functional hepatocyte-like cells in vitro via co-culture with MLSgt20 cells, thus combining the two abovementioned culture techniques. 


\section{Materials and Methods}

\section{Cell Culture}

The MLSgt20 cells were cultured in Dulbecco's modified Eagle's medium

(DMEM) supplemented with10\% Fetal bovine serum (FBS; JRH biosciences, Lenexa,

KS), $1 \mathrm{mM}$ sodium pyruvate (Sigma-Aldrich, St. Louis, MO), $10 \mathrm{mM}$ nicotinamide

(Sigma-Aldrich), 2 mM L-ascorbic acid phosphate (Wako Pure Chemical, Osaka,

Japan), insulin-transferin-selenium supplement (Gibco, Grand Island, NY), $0.1 \mu \mathrm{M}$

dexamethasone (Sigma-Aldrich), and $20 \mathrm{ng} / \mathrm{ml}$ hepatocyte growth factor (HGF; R\&D

Systems Inc., Minneapolis, MN) on collagen type I-coated dishes (AGC techno glass,

Chiba, Japan). The transgenic hESCs that expressed EGFP under the control of human

AFP enhancer/promoter were originally derived from the KhES3 line and were cultured

on a feeder layer of mouse embryonic fibroblasts as described previously (Ishii,

Fukumitsu, Yasuchika, Adachi, Kawase, Suemori, Nakatsuji, Ikai and Uemoto, 2008,

Suemori, et al., 2006).

Differentiation into hepatocyte-like cells 
To differentiate hESCs into endodermal cells, hESCs were cultured as

described previously (Ishii, Fukumitsu, Yasuchika, Adachi, Kawase, Suemori, Nakatsuji, Ikai and Uemoto, 2008). In brief, the hESCs were transferred to Matrigel-coated dishes and cultured in PRMI1640-based medium with $100 \mathrm{ng} / \mathrm{ml}$ activin A (R\&D Systems Inc.) for four days and subsequently with $20 \mathrm{ng} / \mathrm{ml} \mathrm{HGF}$ for five days. The differentiated cells were dissociated with $0.05 \%$ trypsin (Gibco) and EDTA (Dojindo Laboratories, Kumamoto, Japan) solution, followed by resuspension in Hank's balanced salt solution supplemented with 3\% FBS. The EGFP-positive AFP-producing cells were separated using a FACSVantage SE (BD Biosciences, Franklin Lakes, NJ). In order to form cell aggregates, the sorted EGFP-positive cells were either cultured alone or co-cultured with the dissociated MLSgt20 cells in a 1:1 mixture in Sumilon Celltight Spheroid 96-well plates (Sumitomo Bakelite Co., Ltd., Tokyo, Japan) at a density of $1 \times$ $10^{4}$ cells per well in DMEM supplemented with $10 \%$ FBS, $1 \mathrm{mM}$ sodium pyruvate, 10 $\mathrm{mM}$ nicotinamide, $0.2 \mathrm{mM}$ L-ascorbic acid phosphate, insulin-transferin-selenium supplement, $0.1 \mu \mathrm{M}$ dexamethasone, $20 \mathrm{ng} / \mathrm{ml} \mathrm{HGF}$, and $10 \mathrm{ng} / \mathrm{ml}$ oncostatin $\mathrm{M}$ (R\&D Systems Inc.) for seven days after the cell sorting. Correspondingly, the undifferentiated 
hESCs were dissociated using $0.05 \%$ trypsin and EDTA solution and subsequently

co-cultured in a 1:1 mixture with the MLSgt20 cells to generate cell aggregates at the same cell density. The dissociated MLSgt20 cells were also cultured to form cell aggregates. These spheroids were inoculated into 1:80 diluted Matrigel (BD Biosciences) coated-dishes at a density of 6 to 8 spheroids $/ \mathrm{cm}^{2}$ and then were cultured for 14 days. Flow diagrams of the culture protocol are summarized in Fig. 1.

\section{Immunostaining}

The cultured cells were fixed and stained as previously described (Ishii,

Fukumitsu, Yasuchika, Adachi, Kawase, Suemori, Nakatsuji, Ikai and Uemoto, 2008,

Ishii, Yasuchika, Fujii, Hoppo, Baba, Naito, Machimoto, Kamo, Suemori, Nakatsuji and Ikai, 2005). The primary antibodies used in the present study were as follows:

anti-human AFP (Dako, 1:200), anti-human albumin (Bethyl, Montogomery, TX, 1:200), anti-human CYP3A4/7 (Enzo Life Science, Plymouth Meeting, PA, 1:500), and anti-mouse gp38 (a kind gift of Dr. Andrew G. Farr, University of Washington School of Medicine, Seattle, WA, 1:10 dilution). 


\section{Reverse Transcriptase-Polymerase Chain Reaction (RT-PCR)}

An RT-PCR analysis was performed as described previously (Ishii, Fukumitsu,

Yasuchika, Adachi, Kawase, Suemori, Nakatsuji, Ikai and Uemoto, 2008, Ishii,

Yasuchika, Fujii, Hoppo, Baba, Naito, Machimoto, Kamo, Suemori, Nakatsuji and Ikai,

2005, Ishii, et al., 2007). Primers were generated for the following genes: human Nanog,

human Oct-3/4, human GATA4, human AFP, human albumin, human tyrosine

aminotransferase (TAT), human tryptophan-2,3-dioxgenase (TO), human cytochrome

P450 3A4/7 (CYP3A4/7), and glyceraldehyde-3-phosphate dehydrogenase (GAPDH) of

human and mouse genes. The primer sequences and PCR conditions are summarized in

\section{Table 1.}

\section{Hepatocyte Function Analyses}

In order to examine hepatocyte-related functions, the ammonia removal

ability, CYP3A4/7 activity, and the ability to synthesize and store glycogen were

analyzed. The ammonia concentrations in the media after 24-hour cultures were 
measured as described previously (Ishii, Yasuchika, Fujii, Hoppo, Baba, Naito,

Machimoto, Kamo, Suemori, Nakatsuji and Ikai, 2005). CYP3A4/7 activity was

examined using P450-Glo CYP3A4 Assays (Luciferin-PFBE; Promega, WI, USA)

according to the manufacturer's protocol. For CYP3A4/7 induction studies, cultured

cells were treated in the presence of $50 \mu \mathrm{M}$ dexamethasone for 48 hours. The

dexamethasone was dissolved with 99.5\% ethanol. Huh7 (American Type Culture

Collection, Manassas, VA), a human hepatocellular carcinoma cell line, was used as a

control. Periodic acid-Shiff (PAS) staining was performed to detect the presence of

intracellular glycogen, according to the established standard protocol (Hoppo, Fujii,

Hirose, Yasuchika, Azuma, Baba, Naito, Machimoto and Ikai, 2004, Ishii, Yasuchika,

Fujii, Hoppo, Baba, Naito, Machimoto, Kamo, Suemori, Nakatsuji and Ikai, 2005).

\section{Statistical analysis}

All experiments were repeated at least three times. The results are presented as the mean $\pm \mathrm{SD}$. The statistical analyses were performed using Student's $t$ - or

Dunnett's test. A $P$ value of $<0.05$ was considered to be statistically significant. 


\section{Results}

\section{Formation of cell aggregates}

Spheroid cultures were utilized to mature hESCs into functional hepatocytes.

Although the MLSgt20 cells could form cell spheroids (Fig. 2a), the sorted

EGFP-positive AFP-producing cells per se did not form cell aggregates in the absence of MLSgt20 cells. The trypsinized undifferentiated hESCs did not form cell aggregates in the absence of MLSgt20 cells either (data not shown). However, these cells could form spherical cell aggregates in the presence of MLSgt20 cells (Fig. 2b, c). These spheroids were placed on Matrigel-coated dishes and some of them spread throughout the culture dishes (Fig. 2e, f). The spreading cells formed a monolayer that was morphologically identical or very similar to MLSgt20 cells (Fig. 2d, g). Among these monolayer cells, there were piled-up colonies consisting of polygonal hepatocyte-like cells (Fig. 2h).

Immunocytological characteristics of the hESC-derived hepatocyte-like cells and MLSgt20 cells 
Immunocytological assays were performed in order to characterize the

differentiated cells. Immunostaining revealed that the spreading monolayer cells did not express human AFP, albumin, or CYP3A4/7 (Fig. 3a-c). The cells expressed murine gp38, which was one of the cell surface markers of the MLSgt20 cells (Fig. 3d). However, the piled-up colonies of the co-cultured hESC-derived AFP-producing cells expressed human AFP (Fig. 3e) and albumin (Fig. 3f), and some cells were binucleate (Fig. 3g). In contrast, these colonies did not express murine gp38 (Fig. 3i). Less than $10 \%$ of the piled-up cells expressed CYP3A4/7 (Fig. 3h). These results suggested that the MLSgt20 cells predominantly proliferated and formed a monolayer surrounding the hESC-derived colonies. Although all the colonies expressed AFP and/or albumin, some colonies primarily expressed AFP, while some primarily expressed albumin, and others expressed both (Fig. 3j). Therefore, the co-cultured hESCs were heterogeneous cell populations at various stages of differentiation.

\section{Hepatocyte-related marker expression}

RT-PCR revealed that the hESC-derived AFP-producing cells co-cultured 
with the MLSgt20 cells expressed some mature hepatocyte markers, including albumin,

TAT, TO, and CYP3A4/7 (Fig. 4). Although their expression pattern was similar to that of adult livers, the co-cultured hESC-derived AFP-producing cells still expressed AFP and GATA4, unlike the adult livers. These results were consistent with the immunocytological observations mentioned above. However, the undifferentiated hESCs co-cultured with MLSgt20 cells did not express the hepatocyte-related markers, with the exception of weak expressions of AFP and TAT, whereas they still expressed undifferentiated ESC markers, including Nanog and Oct-3/4.

\section{Hepatocyte function analyses}

The ammonia removal ability (Fig. 5a), CYP3A4/7 activity (Fig. 5b), and glycogen synthesis and storage ability $(\mathbf{F i g} . \mathbf{5 c}, \mathbf{d})$ were measured in order to evaluate the extent of the hepatocyte functions. The hESC-derived AFP-producing cells co-cultured with MLSgt20 cells had a significantly greater ability to remove ammonia than the MLSgt20 cells alone or the undifferentiated hESCs co-cultured with MLSgt20 cells. Furthermore, the hESC-derived AFP-producing cells co-cultured with MLSgt20 
cells displayed a higher CYP3A4/7 activity than the other cell groups, even in the absence of dexamethasone-mediated CYP3A4/7 induction. The CYP3A4/7 activity of the hESC-derived AFP-producing cells co-cultured with MLSgt20 cells displayed an approximately 1.45 -fold increase by dexamethasone with statistical significance. However, an extended culture period did not increase the degree of CYP3A4/7 activity (Supplementary Fig. 1). The Huh7 cells showed a lower level of CYP3A4/7 activity than the co-cultured hESC-derived AFP-producing cells. In addition, dexamethasone did not increase the CYP3A4/7 activity of Huh7. Moreover, the piled-up colonies of the co-cultured hESC-derived AFP-producing cells were positive for PAS staining, while the flat monolayer cells, which were morphologically supposed to be MLSgt20 cells, were not stained by PAS staining. These results indicated that the hESC-derived AFP-producing cells co-cultured with MLSgt20 cells possessed the ability to produce and store glycogen. 


\section{Discussion}

The maturation of murine fetal hepatoblasts into hepatocytes required direct

contact with mesenchymal cells residing in fetal livers (Hoppo, Fujii, Hirose, Yasuchika,

Azuma, Baba, Naito, Machimoto and Ikai, 2004, Kamo, Yasuchika, Fujii, Hoppo,

Machimoto, Ishii, Fujita, Tsuruo, Yamashita, Kubo and Ikai, 2007). The mesenchymal

cells that accelerated the hepatic cell maturation were defined as the

CD45-CD49f \pm Thy $1+$ gp 38+ cell fraction. Based on these findings, the MLSgt20 cell

line was established from this cell fraction by transfection of the SV40 large T antigen

gene (Fukumitsu, Ishii, Yasuchika, Amagai, Saitoh, Kawamoto, Kawase, Suemori,

Nakatsuji, Ikai and Uemoto, 2009). Co-culture with the MLSgt20 cell line promoted the

hepatic maturation of murine hepatic progenitor cells as well as murine ESC-derived

AFP-producing endodermal cells. The use of media conditioned with the MLSgt20 cells

had no effect on hepatic maturation. Because it was unclear whether the MLSgt20 cells

affected the hepatic maturation of hESCs, we sought to establish a method to mature

hESCs into functional hepatocytes using MLSgt20 cells.

It was technically difficult to culture hESC-derived cells after cell sorting 
because hESCs were vulnerable to trypsin treatment (Hasegawa, et al., 2007, Suemori, Yasuchika, Hasegawa, Fujioka, Tsuneyoshi and Nakatsuji, 2006). However, co-culture with MLSgt20 cells enabled the sorted hESC-derived AFP-producing cells to survive and form cell aggregates. Furthermore, the co-cultured hESC-derived AFP-producing cells expressed hepatic markers and hepatocyte functions, including ammonia removal ability and glycogen synthesis/storage capability. Moreover, dexamethasone induced the CYP3A4/7 activity of the co-cultured cells. Although the Huh7 cells also displayed CYP3A4/7 activity, it was not induced by dexamethasone. These results indicated that the co-cultured hepatocyte-like cells derived from hESCs displayed physiological metabolic reactions. However, the results of immunostaining and RT-PCR revealed that the co-cultured cells were at various stages of differentiation and varied from the AFP-producing hepatoblast-like immature cells to the CYP3A4/7-expressing mature hepatocyte-like cells. Further research is necessary to obtain a homogeneous functional hepatocyte culture from hESCs. The undifferentiated hESCs did not express any mature hepatic markers or hepatocyte functions even after co-culture with MLSgt20 cells. These findings indicate that MLSgt20 cells can induce the maturation of committed 
progenitors into hepatocytes, but they cannot directly specify the endodermal fate of hESCs.

CYP3As are the most abundant CYP enzymes in the human liver, and are involved in the metabolism of 45-60\% of currently used drugs (Wilkinson, 2005, Wojnowski, 2004). There are four isoforms in the CYP3A subfamily, namely CYP3A4, CYP3A5, CYP3A7, and CYP3A43 (Finta and Zaphiropoulos, 2000, Gellner, et al., 2001, Westlind, et al., 2001). CYP3A4 is the major CYP3A isoform in the human adult liver (Stevens, 2006). However, CYP3A7 is the major component of fetal hepatic CYP enzymes, and this isoform switches to CYP3A4 several weeks after birth (Lacroix, et al., 1997). Although the induction of CYP3A4 is mediated by ligands for the pregnane $X$ receptor, constitutive andorostane receptor, and glucocorticoid receptor (GR), CYP3A7 induction is primarily mediated by the GR pathway, thus suggesting that this isoform plays a role in fetal steroid metabolism and homeostasis (Matsunaga, et al., 2004). In this study, it was technically impossible to distinguish between CYP3A4 and CYP3A7 because of the lack of convenient assays to distinguish the CYP3A isoforms.

In conclusion, hESCs successfully matured into functional hepatocyte-like 
cells in vitro via co-culturing with the fetal murine hepatic stromal cell line, MLSgt20.

The hESC-derived hepatocyte-like cells displayed hepatocyte functions, particularly

CYP3A4/7 activity and the dexamethasone-mediated CYP3A4/7 induction. Our results

might therefore positively contribute to the advancement of cell transplantation therapy and the development of bio-artificial livers, as well as the prediction of the drug response and safety in drug discovery systems in the future. 


\section{Acknowledgements}

We thank Dr. Andrew G. Farr for providing the anti-gp38 antibodies. This

work was supported in part by grants from the New Energy and Industrial Technology

Development Organization (NEDO) and the Scientific Research Fund of the Ministry of

Education, Culture, Sports, Science, and Technology of Japan. 


\section{References}

Cai J, Zhao Y, Liu Y, Ye F, Song Z, Qin H, Meng S, Chen Y, Zhou R, Song X, Guo Y, Ding M, Deng H (2007) Directed differentiation of human embryonic stem cells into functional hepatic cells. Hepatology 45:1229-1239

Chen SS, Fitzgerald W, Zimmerberg J, Kleinman HK, Margolis L (2007) Cell-cell and cell-extracellular matrix interactions regulate embryonic stem cell differentiation. Stem Cells 25:553-561

D'Amour KA, Agulnick AD, Eliazer S, Kelly OG, Kroon E, Baetge EE (2005) Efficient differentiation of human embryonic stem cells to definitive endoderm. Nat Biotechnol 23:1534-1541

Duan Y, Catana A, Meng Y, Yamamoto N, He S, Gupta S, Gambhir SS, Zern MA (2007) Differentiation and enrichment of hepatocyte-like cells from human embryonic stem cells in vitro and in vivo. Stem Cells 25:3058-3068

Ek M, Soderdahl T, Kuppers-Munther B, Edsbagge J, Andersson TB, Bjorquist P, Cotgreave I, Jernstrom B, Ingelman-Sundberg M, Johansson I (2007) Expression of drug metabolizing enzymes in hepatocyte-like cells derived from human embryonic stem cells. Biochem Pharmacol 74:496-503

Finta C, Zaphiropoulos P (2000) The human cytochrome P450 3A locus. Gene evolution by capture of downstream exons. Gene 260:13-23

Fukumitsu K, Ishii T, Yasuchika K, Amagai Y, Saitoh M, Kawamoto T, Kawase E, Suemori H, Nakatsuji N, Ikai I, Uemoto S (2009) Establishment of a cell line derived from mouse fetal liver that have the character to promote the hepatic maturation of mouse embryonic stem cells by co-culture method. Tissue Eng Part A

Gellner K, Eiselt R, Hustert E, Arnold H, Koch I, Haberl M, Deglmann C, Burk O, Buntefuss D, Escher S, Bishop C, Koebe H, Brinkmann U, Klenk H, Kleine K, Meyer U, Wojnowski L (2001) Genomic organization of the human CYP3A locus: identification of a new, inducible CYP3A gene. Pharmacogenetics 11:111-121

Hasegawa K, Cowan AB, Nakatsuji N, Suemori H (2007) Efficient multicistronic expression of a transgene in human embryonic stem cells. Stem Cells 25:1707-1712

Hoppo T, Fujii H, Hirose T, Yasuchika K, Azuma H, Baba S, Naito M, Machimoto T, 
Ikai I (2004) Thy1-positive mesenchymal cells promote the maturation of CD49f-positive hepatic progenitor cells in the mouse fetal liver. Hepatology 39:1362-1370

Ishii T, Fukumitsu K, Yasuchika K, Adachi K, Kawase E, Suemori H, Nakatsuji N, Ikai I, Uemoto S (2008) Effects of extracellular matrixes and growth factors on the hepatic differentiation of human embryonic stem cells. Am J Physiol Gastrointest Liver Physiol 295:G313-321

Ishii T, Yasuchika K, Fujii H, Hoppo T, Baba S, Naito M, Machimoto T, Kamo N, Suemori H, Nakatsuji N, Ikai I (2005) In vitro differentiation and maturation of mouse embryonic stem cells into hepatocytes. Exp Cell Res 309:68-77

Ishii T, Yasuchika K, Machimoto T, Kamo N, Komori J, Konishi S, Suemori H, Nakatsuji N, Saito M, Kohno K, Uemoto S, Ikai I (2007) Transplantation of embryonic stem cell-derived endodermal cells into mice with induced lethal liver damage. Stem Cells 25:3252-3260

Kamo N, Yasuchika K, Fujii H, Hoppo T, Machimoto T, Ishii T, Fujita N, Tsuruo T, Yamashita JK, Kubo H, Ikai I (2007) Two populations of Thy1-positive mesenchymal cells regulate in vitro maturation of hepatic progenitor cells. Am J Physiol Gastrointest Liver Physiol 292:G526-534

Lacroix D, Sonnier M, Moncion A, Cheron G, Cresteil T (1997) Expression of CYP3A in the human liver--evidence that the shift between CYP3A7 and CYP3A4 occurs immediately after birth. Eur J Biochem 247:625-634

Matsunaga T, Maruyama M, Harada E, Katsuyama Y, Sugihara N, Ise H, Negishi N, Ikeda U, Ohmori S (2004) Expression and induction of CYP3As in human fetal hepatocytes. Biochem Biophys Res Commun 318:428-434

Schuldiner M, Yanuka O, Itskovitz-Eldor J, Melton DA, Benvenisty N (2000) Effects of eight growth factors on the differentiation of cells derived from human embryonic stem cells. Proc Natl Acad Sci U S A 97:11307-11312

Stevens J (2006) New perspectives on the impact of cytochrome P450 3A expression for pediatric pharmacology. Drug Discov Today 11:440-445

Suemori H, Yasuchika K, Hasegawa K, Fujioka T, Tsuneyoshi N, Nakatsuji N (2006) Efficient establishment of human embryonic stem cell lines and long-term maintenance with stable karyotype by enzymatic bulk passage. Biochem Biophys Res Commun 345:926-932 
Westlind A, Malmebo S, Johansson I, Otter C, Andersson T, Ingelman-Sundberg M, Oscarson M (2001) Cloning and tissue distribution of a novel human cytochrome p450 of the CYP3A subfamily, CYP3A43. Biochem Biophys Res Commun 281:1349-1355

Wilkinson G (2005) Drug metabolism and variability among patients in drug response. N Engl J Med 352:2211-2221

Wojnowski L (2004) Genetics of the variable expression of CYP3A in humans. Ther Drug Monit 26:192-199 


\section{Figure Legends}

Fig. 1

Summary of the culture protocol.

\section{Fig. 2}

Morphological features of the cultured cells. Fig. 2a-c show the cell cultures five days after cell sorting, and Fig. 2d-h show the morphology 21 days after cell sorting. The MLSgt 20 cells cultured alone (a, d, and g), the hESC-derived AFP-producing cells co-cultured with the MLSgt20 cells (b, e, and h), and the undifferentiated hESCs co-cultured with the MLSgt20 cells ( $\mathrm{c}$ and f) formed cell aggregates and extended over Matrigel-coated dishes. The area encircled by the dashed line in Fig. $2 \mathbf{h}$ indicates a piled-up colony consisting of large, dark, polygonal cells. Scale bars: (a-c), $150 \mu \mathrm{m}$; (d-f), $400 \mu \mathrm{m} ;(\mathrm{g}, \mathrm{h}), 50 \mu \mathrm{m}$.

\section{Fig. 3}

Immunostaining of the MLSgt20 cells cultured alone (a-d) and the hESC-derived AFP-producing cells co-cultured with the MLSgt20 cells (e-j). The MLSgt20 cells 
cultured alone were negative for human AFP, albumin (Alb), or CYP3A4/7 (a-c) and positive for murine gp38 (d). The piled-up colonies in the culture of the hESC-derived AFP-producing cells with the MLSgt20 cells were positive for AFP (e), albumin (f, g), and CYP3A4/7 (h) and negative for gp38 (i). Some of these albumin-positive cells were binucleate (g; arrow heads). The area encircled by the dashed line in Fig. 3i indicates the piled-up colony. Green fluorescence indicates the expression of AFP, and red fluorescence indicates albumin in Fig. 3j. Blue fluorescence indicates DAPI. Scale bars: (a-f, h-j), $100 \mu \mathrm{m} ;(\mathrm{g}), 50 \mu \mathrm{m}$.

\section{Fig. 4}

An RT-PCR analysis of the hESC-derived functional hepatocyte-like cells. Total RNA was extracted from the MLSgt20 cells cultured alone (lane 1), the undifferentiated hESCs co-cultured with the MLSgt20 cells (lane 2), the hESC-derived AFP-producing cells co-cultured with the MLSgt20 cells (lane 3), and an adult human liver (lane 4). Nanog and Oct-3/4 served as undifferentiated ESC markers. GATA4 and AFP served as immature hepatocyte markers. Albumin, TAT, TO and CYP3A4/7 served as mature 
hepatocyte markers. GAPDH was used as an internal control, and -RT served as a negative control.

Fig. 5

Hepatocyte function analyses. The white bars indicate the MLSgt20 cells cultured alone, the dotted bars represent the undifferentiated hESCs co-cultured with the MLSgt20 cells, the black bars represent the hESC-derived AFP-producing cells co-cultured with the MLSgt20 cells, and the gray bars represent the Huh7 cells. (a) Ammonia removal abilities were quantified as a percentage of the decreased ammonia concentration after 24-hour culture. The statistical analysis was performed by Dunnett's test. ${ }^{*}, P<0.05$. (b) CYP3A4/7 activities were measured in the absence $(0.1 \mu \mathrm{M}$ dexamethasone $)$ or presence of dexamethasone-mediated induction ( $50 \mu \mathrm{M}$ dexamethasone). ${ }^{*}, P<0.05$ by Student's $t$-test. (c, d) The piled-up colonies of the hESC-derived AFP-producing cells co-cultured with MLSgt20 cells were positive for PAS staining, whereas the surrounding monolayer cells were negative for PAS staining. Scale bars: (c), $400 \mu \mathrm{m}$; (d), $50 \mu \mathrm{m}$. 
Fig. 1, T. Ishii et al.

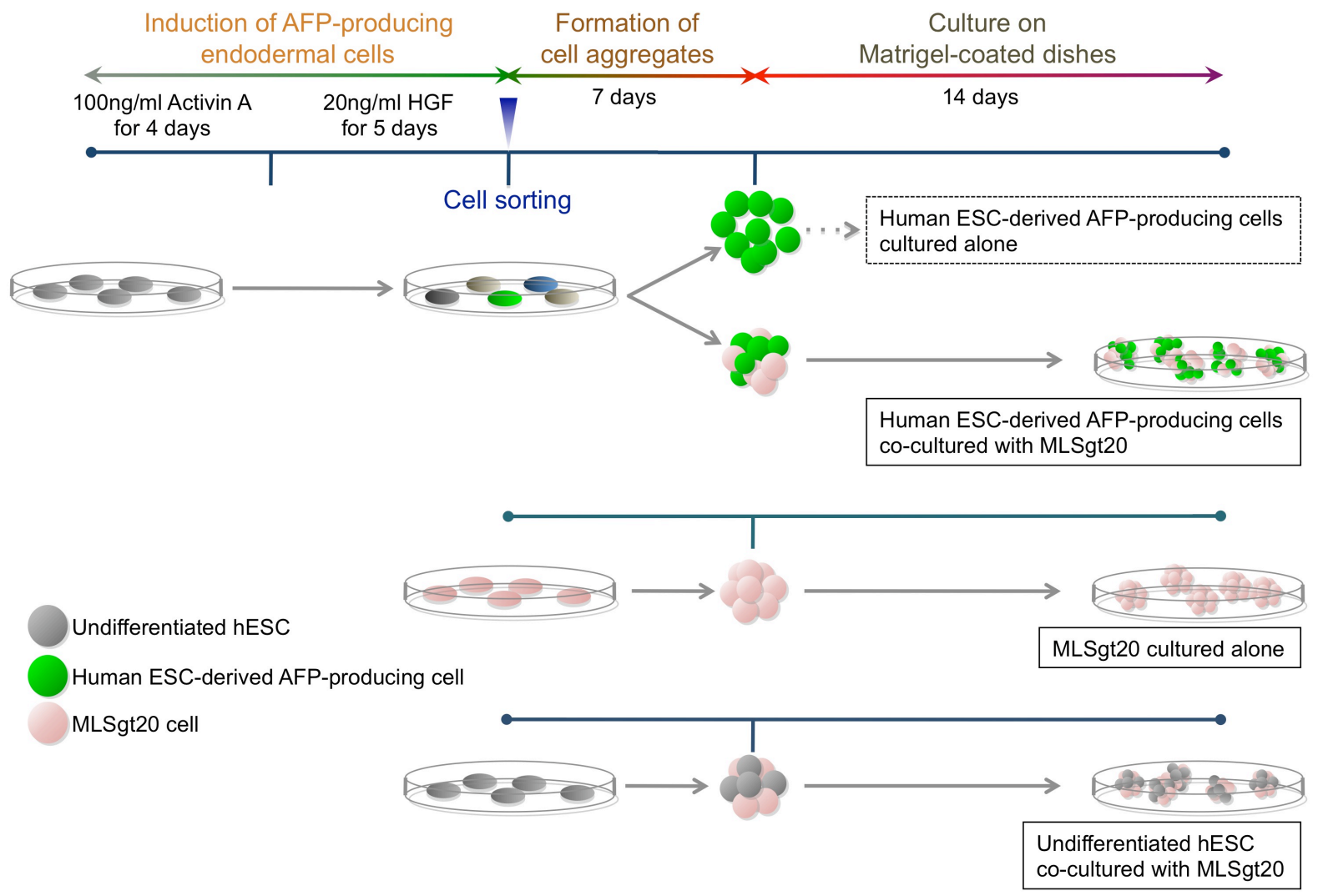


Fig. 2, T. Ishii et al.

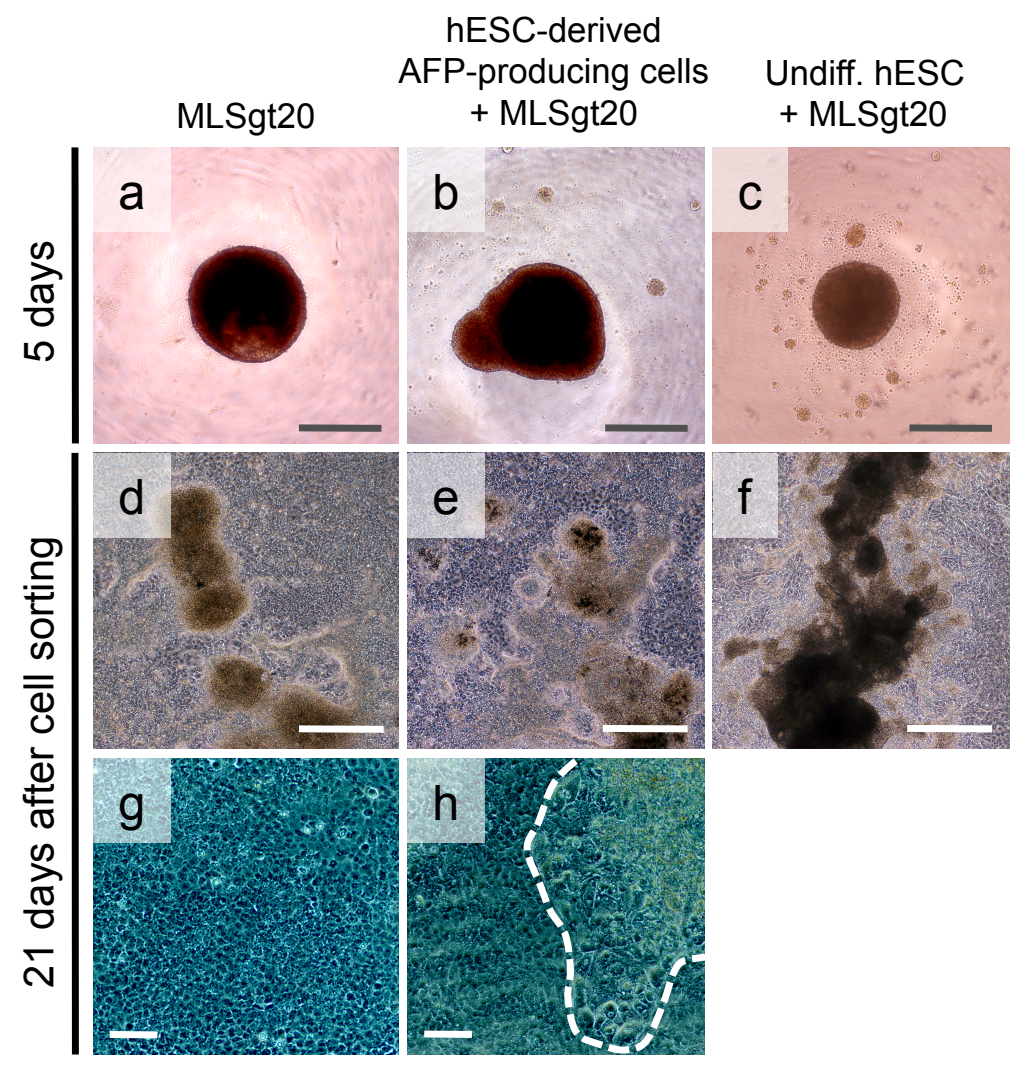


Fig. 3, T. Ishii et al.

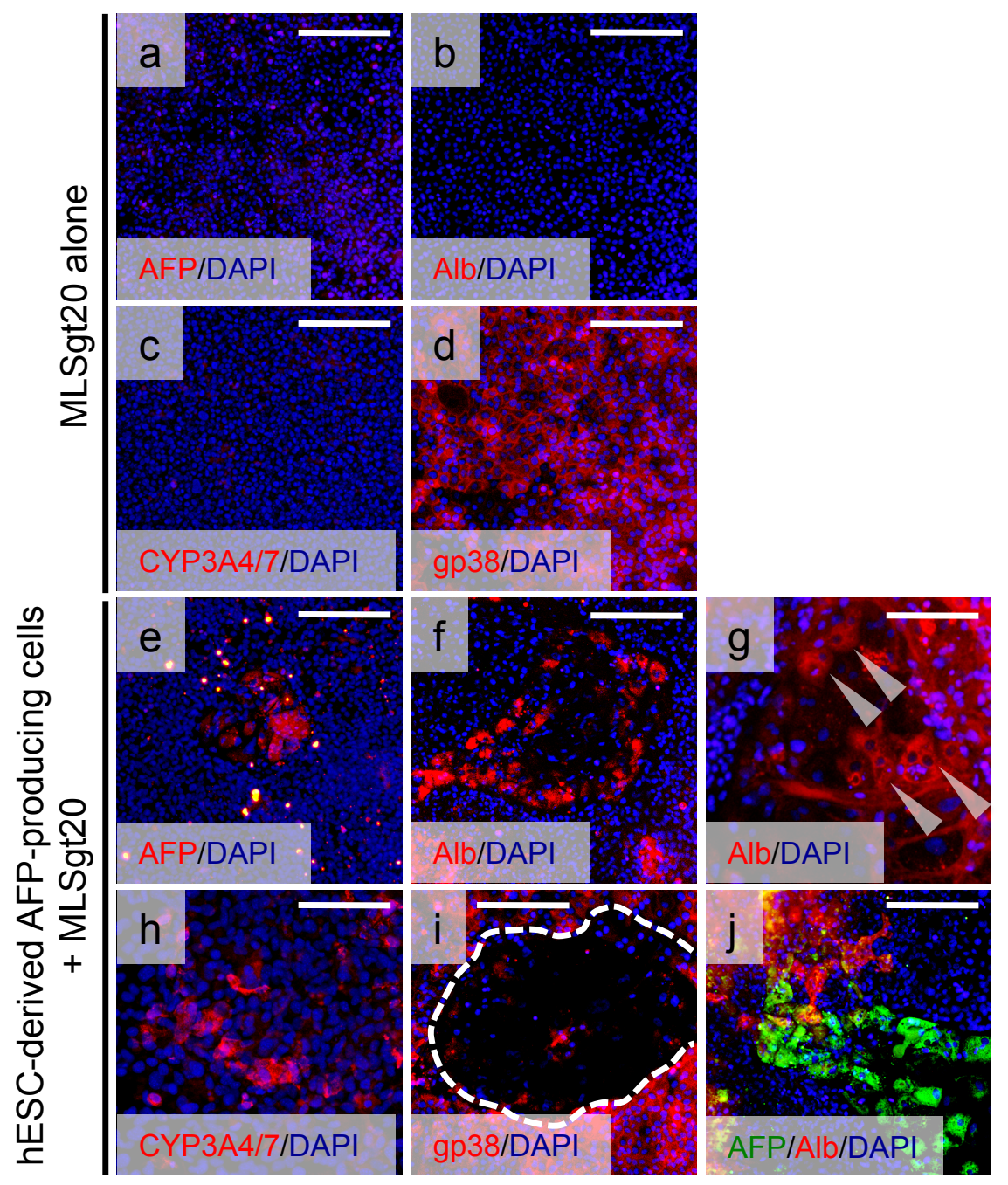


Fig. 4, T. Ishii et al.

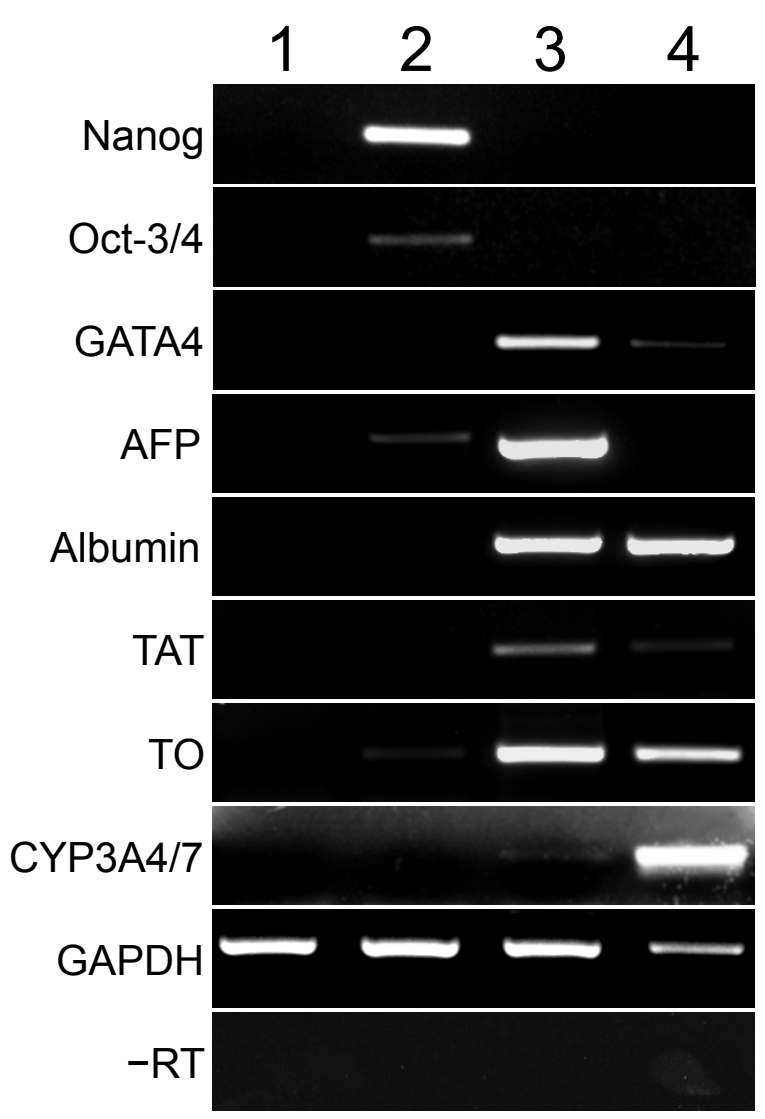


Fig. 5, T. Ishii et al.

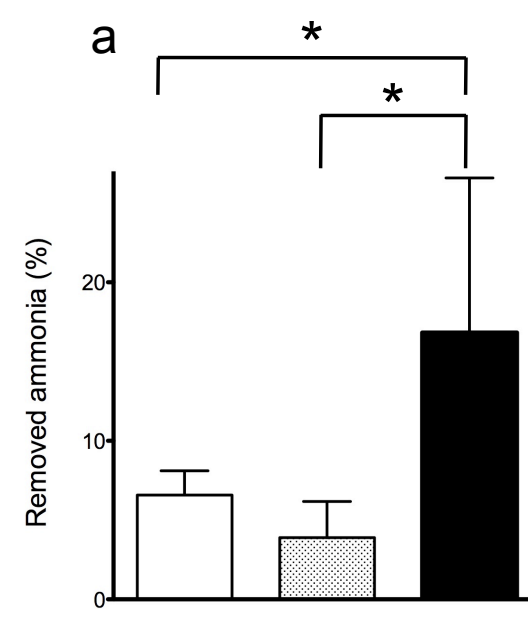

b

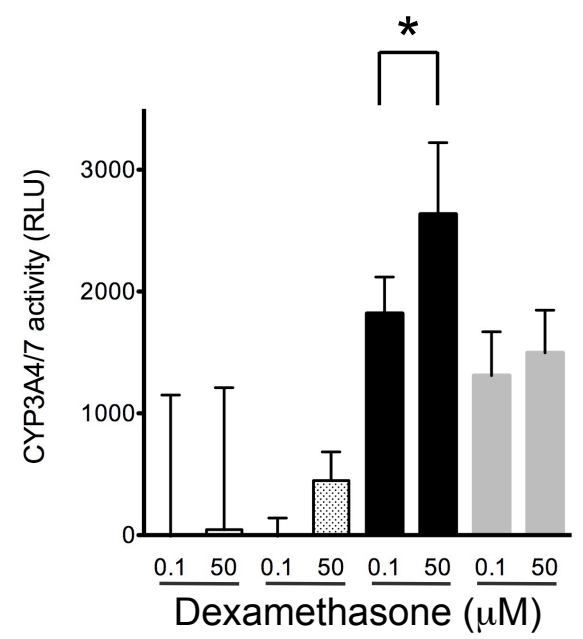

$\square$ MLSgt20 alone

. Undifferentiated hESCs + MLSgt20

hESC-derived AFP-producing cells + MLSgt20

Huh7
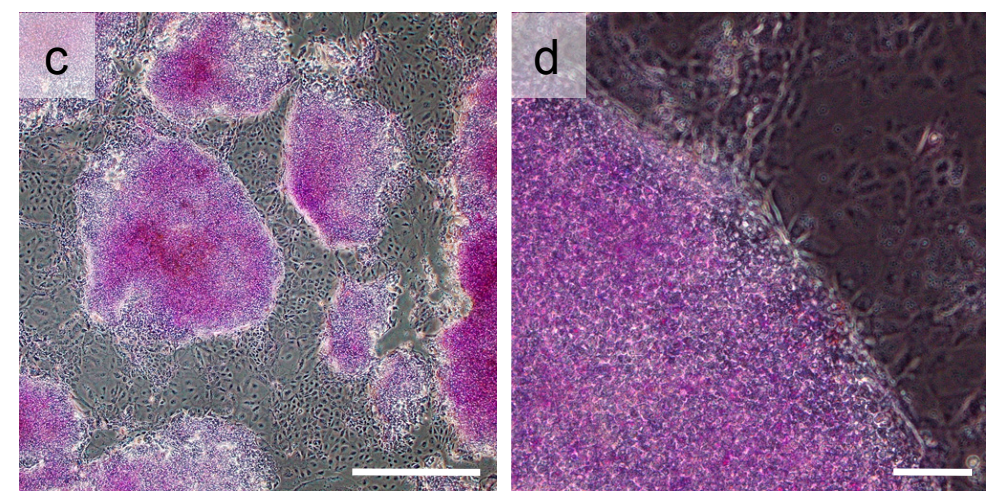